\title{
Investigations of Spectroscopic Factors and Sum Rules from the Single Neutron Transfer Reaction $\left.{ }^{111} \mathbf{C d}(\overrightarrow{\mathbf{d}}, p)\right)^{112} \mathbf{C d}$
}

\author{
D.S. Jamieson ${ }^{1}$,a , P.E. Garrett ${ }^{1}$, G.C. Ball ${ }^{2}$, G.A. Demand ${ }^{1}$, T. Faestermann ${ }^{3}$, P. Finlay ${ }^{1}$, K.L. \\ Green $^{1}$, R. Hertenberger ${ }^{4}$, R. Krücken ${ }^{3}$, K.G. Leach ${ }^{1}$, A.A. Phillips ${ }^{1}$, C.S. Sumithrarachchi ${ }^{1}$, S. \\ Triambak ${ }^{1}$, and H.-F. Wirth ${ }^{4}$ \\ ${ }^{1}$ Department of Physics, University of Guelph, ON, N1G2W1, Canada \\ ${ }^{2}$ TRIUMF, 4004 Wesbrook Mall, Vancouver, British Columbia, V6T 2A3, Canada \\ ${ }^{3}$ Physik Department, Technische Universitat München, D-85748 Garching, Germany \\ ${ }^{4}$ Fakulat für Physik, Ludwig-Maximilians-Universitat Munchen, D-85748m Garching, Germany
}

\begin{abstract}
Cadmium isotopes have been presented for decades as excellent examples of vibrational nuclei, with low-lying levels interpreted as multi-phonon quadrupole, octupole, and mixed-symmetry states. A large amount of spectroscopic data has been obtained through various experimental studies of cadmium isotopes. In the present work, the ${ }^{111} \mathrm{Cd}(\overrightarrow{\mathrm{d}}, \mathrm{p}){ }^{112} \mathrm{Cd}$ reaction was used to investigate the single-particle structure of the ${ }^{112} \mathrm{Cd}$ nucleus. A $22 \mathrm{MeV}$ beam of polarized deuterons was obtained at the Maier-Leibnitz laboratory in Garching, Germany. The reaction ejectiles were momentum analyzed using a Q3D spectrograph, and 130 levels have been identified up to $4.2 \mathrm{MeV}$ of excitation energy. Using DWBA analysis with optical model calculations, spin-parity assignments have been made for observed levels, and spectroscopic factors have been extracted from the experimental angular distributions of differential cross section and analyzing power. In this high energy resolution investigation, many additional levels have been observed compared with the previous (d,p) study using $8 \mathrm{MeV}$ deuterons [1]. There were a total of 44 new levels observed, and the parity assignments of 34 levels were improved.
\end{abstract}

\section{Introduction}

The energy spacings and spin-parity values of low-lying levels in the cadmium isotopes have a compelling similarity to the spectrum of a spherical harmonic vibrational system. Vibrational nuclear structure involves highly collective modes of excitation, where a 1-phonon state is a superposition over many two quasi-particle states near the Fermi surface. Quadrupole and octupole single-phonon and multi-phonon states have been proposed in the cadmium isotopes, as well as coupled quadrupoleoctupole states. The ${ }^{111} \mathrm{Cd}(\overrightarrow{\mathrm{d}}, \mathrm{p}){ }^{112} \mathrm{Cd}$ single neutron transfer reaction probes the single-particle components of states in ${ }^{112} \mathrm{Cd}$, which provides a good test of the assignments made for phonon states within ${ }^{112} \mathrm{Cd}$. The vibrational interpretation of excited states in ${ }^{112} \mathrm{Cd}$ must be consistent with the distribution of single-particle transfer strength among the excited states. A highly collective state, such a multi-phonon excitation, should be weakly populated in a single nucleon transfer.

\footnotetext{
a e-mail: jamiesoa@uoguelph.ca
} 


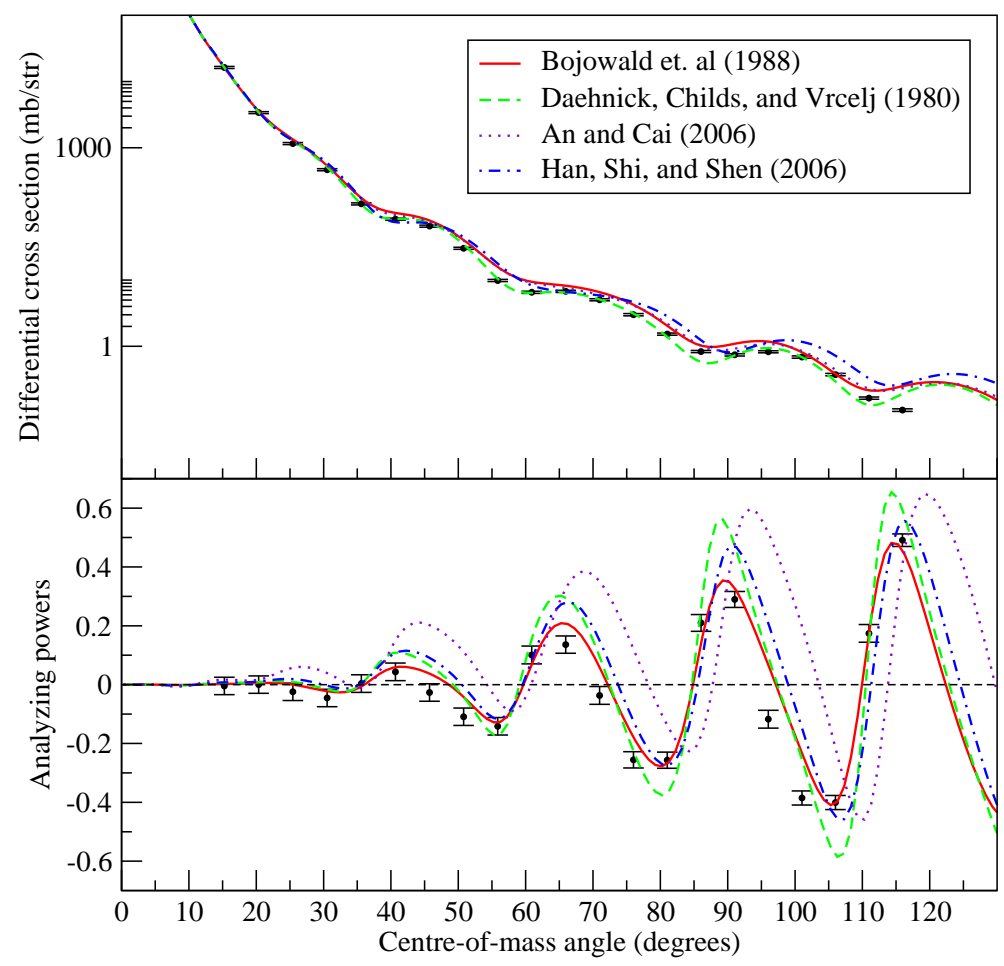

Figure 1: Elastic scattering angular distributions of differential cross section and analyzing power from polarized deuterons on ${ }^{111} \mathrm{Cd}$, with DWBA calculations from global optical model potentials $[2,3,6,7]$.

\section{Spectroscopic factors and sum rules}

A ${ }^{111} \mathrm{Cd}(\vec{d}, \mathrm{p}){ }^{112} \mathrm{Cd}$ single-neutron transfer experiment was conducted at the Maier-Leibniz Laboratory in Germany using an MP tandem Van de Graaff accelerator. Polarized deuterons from Stern-Gerlach ion source were accelerated to $22 \mathrm{MeV}$, and $80 \%$ polarization was achieved. The deuterons impinged on a ${ }^{111} \mathrm{Cd}$ target with a thickness of $150 \mu \mathrm{g} \cdot \mathrm{cm}^{-2}$. Outgoing protons were detected using the Q3D magnetic spectrometer. Spectra were collected from angles ranging from $10^{\circ}$ to $60^{\circ}$ in increments of $5^{\circ}$. Polarized deuteron elastic scattering data were also collected for ${ }^{111} \mathrm{Cd}$ between angles of $15^{\circ}$ and $115^{\circ}$ in $5^{\circ}$ increments.

The angular distributions from the elastic scattering of deuterons on the ${ }^{111} \mathrm{Cd}$ is shown in Figure (1), along with several DWBA calculations using standard global optical model potentials. The deuteron elastic scattering data were used to fix which global optical parameters to use in the DWBA analysis of the transfer data. A sample spectrum from the transfer data is shown in Fig. (2). The potential from reference [3] was chosen. Proton optical model parameters from reference [4] were chosen on the basis of elastic scattering data of protons on ${ }^{112} \mathrm{Cd}$ from the EXFOR database. The proton elastic scattering data was originally published in reference [5]. Adiabatic Distorted Wave Approximation (ADWA) calculations were also used. Both calculations are shown with sample experimental angular distributions of Fig. (3). The DWBA and ADWA calculations were performed using the code FRESCO [8]. 


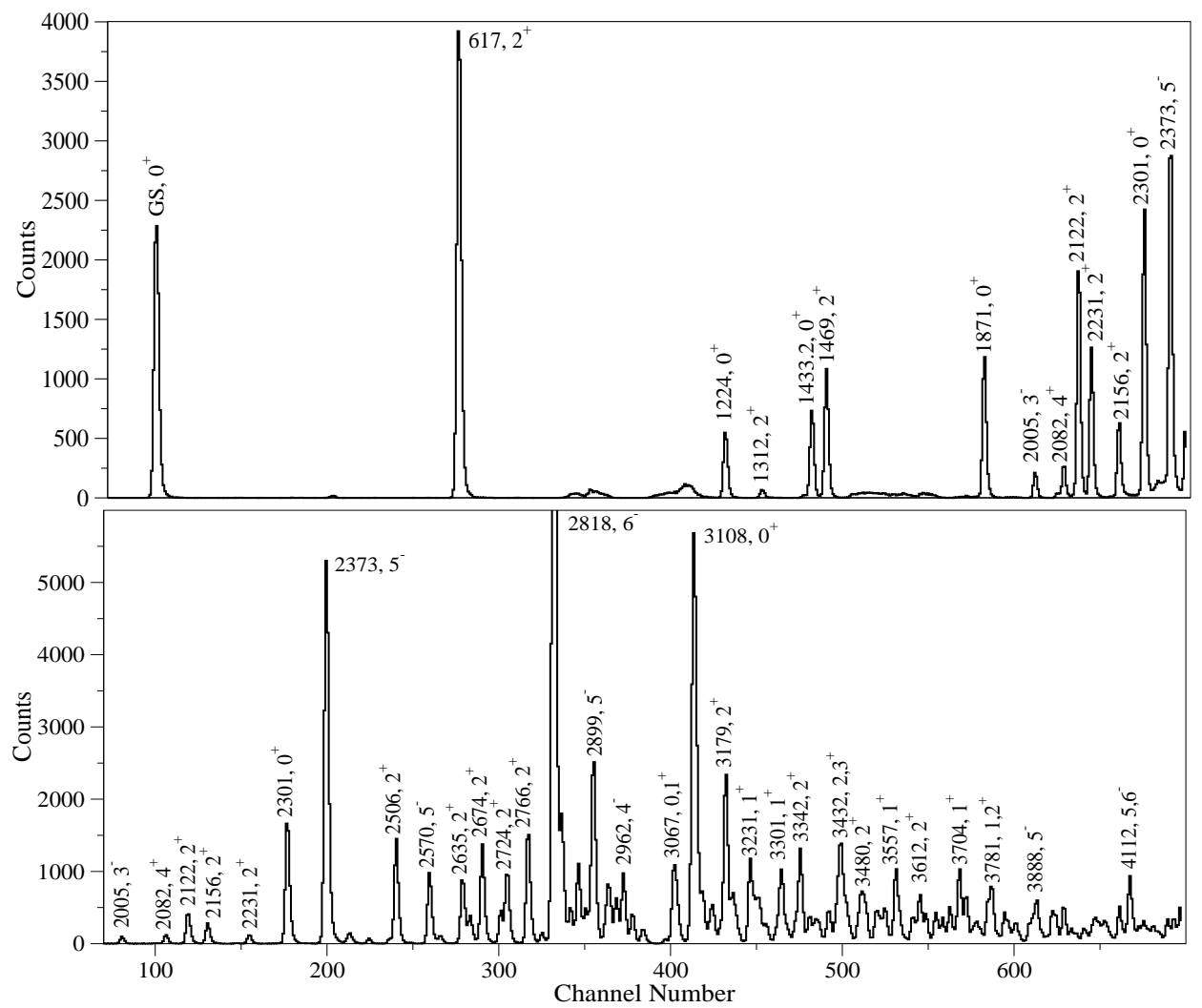

Figure 2: Spectra of the low-lying (top) and high-lying (bottom) excitations in ${ }^{112} \mathrm{Cd}$ from the (d,p) transfer reaction.

The spectra collected during this experimental investigation had energy resolutions of 6-7 keV. The high resolution of the data allowed us to identify 130 levels of ${ }^{112} \mathrm{Cd}$. The angular distributions and analyzing powers, along with DWBA and ADWA calculations, allowed for the identification of the dominant $\ell$ and $j$ involved in the transfer. Where the levels were previously observed in an $\left(n, n^{\prime} \gamma\right)$ experiment [9], the spin-parity of the observed level could be verified, and in some cases made firm. The ground state of the ${ }^{111} \mathrm{Cd}$ target nucleus has $J^{\pi}=1 / 2^{+}$. There were 44 new levels observed in this experiment, and parity assignments were improved for 34 levels.

Sum rules for spectroscopic factors were investigated using results from a previous study of the ${ }^{111} \mathrm{Cd}(\overrightarrow{\mathrm{d}}, \mathrm{t}){ }^{110} \mathrm{Cd}$ reaction [10]. Our total observed $\ell=0$ strength from the $(\mathrm{d}, \mathrm{p})$ transfers was $\sim$ 1.4. The observed $\ell=0$ strength from the $(\mathrm{d}, \mathrm{t})$ pick-up was $\sim 1.1$. According to the sum rules, the total strength from these reactions should be equal to $2 j+1$. The results differ with the sum rule by about $25 \%$, but the two values agree well since the choice of optical potential for the DWBA analysis introduces about a $30 \%$ systematic uncertainty. Spectroscopic factors from the ADWA calculations differed from ones obtained with DWBA calculation by about $20 \%$ on average, so the dominant uncertainty is due to choice of parameters rather than choice of reaction model. The ADWA calculations generally, but with some exceptions, reproduced the transfer angular distributions better than the DWBA calculations. Most of the other $\ell$ transfers add to much less than the sum rule predicts. This indicates that much of the strength for these other $\ell$ transfers is fragmented above $4.2 \mathrm{MeV}$. 

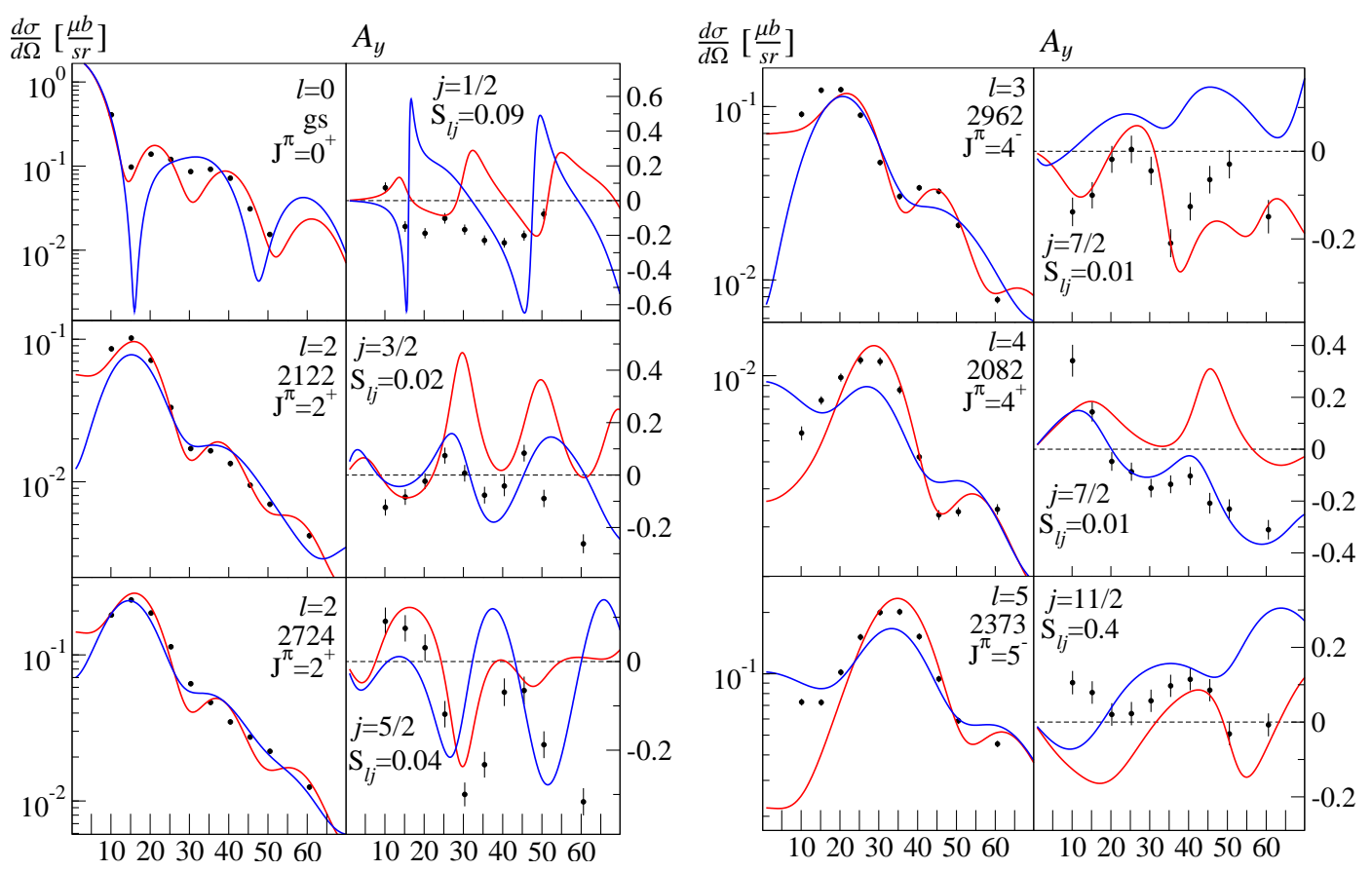

Figure 3: ${ }^{111} \mathrm{Cd}(\vec{d}, \mathrm{p}){ }^{112} \mathrm{Cd}$ angular distributions and analyzing powers with calculations from the FRESCO code. The red curves are ADWA and the blue curves are DWBA calculations. Both curves have been scaled to the experimental data, and the scaling factor $\left(\mathrm{S}_{\ell j}\right)$, is the spectroscopic factor.

\section{Summary and outlook}

Spectroscopic factors have been obtained for 130 populated levels in ${ }^{112} \mathrm{Cd}$ from the (d,p) transfer reaction. Angular distributions of cross sections and analyzing powers from the experiment are well reproduced by the DWBA and ADWA calculations using global OMPs. Spin-parities have been assigned to each observed level. The standard interpretation of ${ }^{112} \mathrm{Cd}$ as a vibrational nucleus will be reassessed in light of the new spectroscopic data from this experiment, including the distribution of single-particle strength for the transfer.

\section{References}

[1] P. D. Barnes, J. R. Comfort, and C.K. Bockelman, Phys. Rev. 155, 1319, (1967).

[2] J. Bojowald et al., Phys. Rev. C 38, 1153 (1988).

[3] W.W. Daehnick, J.D. Childs, and Z. Vrcelj, Phys. Rev. C 21, 2253, (1980).

[4] A.J. Koning and J.P. Delaroche, Nuc. Phys. A 713, 231, (2003).

[5] R.M. Petit et. al., J. Phys. G 20, 1955, (1994).

[6] Haixia An and Chonghai Cai, Phys. Rev. C 73, 054605 (2006).

[7] Y. Han, Y. Shi, and Q. Shen, Phys. Rev. C 74, 044615 (2006).

[8] I.J. Thompson, Comp. Phys. Rep. 7, 167, (1988).

[9] P.E. Garrett et al., Phys Rev. C 64, 024316, (2001).

[10] N. Blasi et al., Nuc. Phys. A 536, 1, (1992). 